\title{
What is learned about nontarget items in simple visual search?
}

\author{
JOHN H. FLOWERS and KAREN L. SMITH \\ University of Nebraska, Lincoln, Nebraska
}

\begin{abstract}
Human sensitivity to correlational structure between nontargets and likelihood of target presence in a visual letter-search task were studied in two experiments. In each of these experiments, the performance of subjects for whom the nontarget information was altered in the final trial block was compared with the performance of subjects for whom the nontarget information did not change. When stimulus strings were presented individually on a computer screen and subjects were required to make a yes-no decision about target presence (Experiment 1), the change in nontarget structure resulted in increased reaction times for target-absent trials. When subjects searched simultaneously for three possible targets (Experiment 2), the change in nontarget structure produced increased error rates and increased reaction times for both target-absent and target-present trials. Correlations between the amount of predictive information in individual stimulus strings and reaction times also showed that both switching and nonswitching subjects were sensitive to the nontarget context. However, neither self-reports of strategy nor postexperiment choices between context-consistent and -inconsistent letter strings indicated any explicit knowledge of the predictive information in the nontarget stimuli. Subjects can thus acquire and benefit from, apparently without awareness, information about subtle correlational structure in nontarget elements in simple visual search.
\end{abstract}

The ability to use predictive context is a key component of efficient performance of many types of perceptual and cognitive tasks. There has recently been a resurgence of interest in the mechanisms involved in learning to use information from prior or coincident perceptual events to enhance performance of a wide range of experimental tasks, including probability learning, sequence learning, simulated control processes, and flanker effects in perceptual classification. In many of these tasks, learning about predictive context appears to be a largely implicit process that is independent of conscious rule-based strategies (e.g., Berry \& Dienes, 1993; Reber, 1993).

One important issue for understanding how these forms of learning about event structure occur is the role of attention in acquiring information about event correlations. In sequence-learning tasks, for example, the nature of the task requires subjects to focus attention on each event. In a flanker task, in contrast, subjects are instructed to focus attention on a specific spatial location to classify a target and to "ignore" flanking nontargets, although correlations between these nontargets and the targets (or perhaps the required responses) will still affect speed of responding to the attended target (Miller, 1987). The nontarget flankers in such tasks are usually few in number (typically one element to the right and one element to the left of the target) and are located in close proximity (typically less than $1^{\circ}$ of visual angle) to the target. Even though subjects may be instructed to "ignore" the flankers, accounts of the spatial

Correspondence concerning this article should be addressed to John H. Flowers or Karen L. Smith, University of Nebraska, Lincoln, Nebraska 68588-0308 (e-mail: jflowers@unl.edu or ksmith@unlgradl. unl.edu). extent of visual attention suggest either that there are limits to its acuity of focus (e.g., B. A. Eriksen \& C. W. Eriksen, 1974; C. W. Eriksen \& St. James, 1986), or that the constraints of the task and display promote less than optimal spatial focusing of attention (Yantis \& Johnston, 1990). Under such conditions, cognitive resources are apparently available to learn and apply information about correlations between target and nontarget elements.

To what extent might such learning occur when the task involves displays of greater complexity, in terms of both heterogeneity of nontarget elements and the number of simultaneously displayed elements? For focusedattention tasks containing multiple elements, in which subjects must identify a target at a cued location, Yantis and Johnston (1990) have shown that influences from nonadjacent distractors can be virtually eliminated. Given that finding, it would seem doubtful that the learning of probability associations between previously unrelated targets and distractors would occur in tasks involving heterogeneous displays that require identification of a target at a known or cued location. However, many "real-world" search tasks, performed by both humans and other animals, involve displays and task demands that fall between those of a simple flanker task and classifying an attentionally cued element in a complex multi-element display.

For such search tasks, perceptual and learning mechanisms that are sensitive to probabilistic contingencies between background features and target likelihood could be advantageous, provided the costs of the required perceptual analysis and storage of nontarget information are sufficiently low. Whether the task involves a human's preparation for responding to certain critical events on a radar display or a bird's decision to peck at a particular 
location on a tree to reveal a prey insect, considerable efficiency might be gained by developing a search "expertise" that takes into account how background elements affect the probability of locating the correct target. On the other hand, for tasks in which target detection can be accomplished by some form of rapid, low-level feature detection, or "search image" matching, little or no gain might be afforded by such contextual processing of the background. For example, in the extreme case of a "pop-out" search task, for which neither the number nor the density of nontargets has any appreciable effect on time to detect the target (Treisman \& Gelade, 1980), it seems highly unlikely that use of information requiring detailed perceptual processing of the background would provide any additional benefit or affect performance. There are a great many natural and laboratory visual search tasks within a range of search "complexity" that fall between pop-out and the need to sequentially scrutinize individual items in an effortful manner. In these tasks, contextual processing of background items may play a significant role.

Furthermore, if background context is important in task performance, it is also interesting to question how it is important. For example, are subjects aware of the influence of the background on their processing? If so, do subjects develop an explicit knowledge or rule about the statistical relationships between background and target presence?

To examine these issues, the present study required subjects to scan for instances of a letter target among strings of relatively heterogeneous nontarget letters. These nontarget letters encompassed a wide range of variation of visual features, some of which were shared with the target letter or letters. Stimulus displays were constructed by a stochastic selection process that was somewhat more likely to choose certain nontarget letters to appear in the same spatial region (within the same six-letter string) in which a target appeared, and more likely to select certain other letters to appear in strings for which a target was absent. Other nontarget letters were equally likely to appear regardless of whether the string contained a target. This procedure thus introduced a relatively subtle correlation between the characteristics of letter distributions and likelihood of encountering a target in the same vicinity. This type of correlation "structure" can be viewed as analogous to correlation structures among natural items located in various ecological niches-for example, a certain species of insect is more likely to be located near certain species of plants, but no plant guarantees the presence or absence of the insect. Furthermore, it is the type of relationship that does not lend itself well to coding as an explicit verbal rule, because it involves multiple stimuli and is nondeterministic (Ericsson \& Simon, 1980).

The influence of this structure on search speed and accuracy of search performance was evaluated in two experiments that varied in task conditions expected to influence the degree of search difficulty and potentially the degree of processing of nontarget items. Both experiments were followed by a questionnaire designed to assess the degree and type of awareness of the relationship between target presence and background context. Experiment 1 in- volved individual presentation of individual letter strings and required a two-choice (yes-no) detection of a single target letter. In Experiment 2, a yes-no task was also used, but it required subjects to search for the occurrence of any of three different target letters. In both experiments, the effects of the correlation bias between targets and nontargets was evaluated by the inclusion of terminal trial blocks in which the letter correlations were "reversed" for one group of subjects, permitting computations of costs in speed and errors relative to those of subjects who did not experience the reversal. Furthermore, the stochastic stimulus-generation process made some target-containing strings have more "predictive" and fewer "counterpredictive" letters than did others. Target-absent strings also varied in the relative numbers of counterpredictive and predictive letters. It was thus possible to examine the influence of the relative numbers of predictive and nonpredictive letters on reaction time to individual trials, even for the subjects who did not have the correlations reversed in the final trial block.

\section{EXPERIMENT 1}

\section{Method}

Subjects. Forty undergraduate students from an introductory psychology course participated in a single experimental session lasting approximately $1 \mathrm{~h}$. For participating, each subject received partial credıt for a course requirement.

Stimulus materials. Stimuli consisted of lists of 80 six-character capital letter strings. The letter strings were generated by a computer program (written in Microsoft QuickBasic Version 4.5) that used the pseudorandom number generator to select the sequence of letters in each string according to the following constraints. First, the list-generation program made a "decision" (with a probability of .5) about whether a letter string would contain the target letter (which was the letter S). On the occasions when a letter string was to contain the target letter, six nontarget letters were chosen with a probability of .53 of a letter's being a "predictive" letter $(C, M$, or $\mathrm{V})$, a probability of .33 of its being a "nonpredictive" letter $(R, O$, or N), and a probability of 13 of its being a "counterpredictive" letter $(\mathrm{J}, \mathrm{W}$, or $\mathrm{Y})$. The program then replaced one of the six letters with the target letter $S$. On the occasions when a target-absent string was to be generated, a string of six nontarget letters was chosen with a .53 probability of the choice's being a "counterpredictive" letter $(\mathrm{J}, \mathrm{W}, \mathrm{Y})$, a .33 probability of its being a "nonpredictive" letter $(\mathrm{N}$, $\mathrm{O}$, or $\mathrm{R})$, and a .13 probability of 1 ts being a "predictive" letter (C, $\mathrm{M}$, or V). Twenty lists of letter strings (Stimulus Set A) were generated using this procedure and 20 lists (Stimulus Set B) were generated using an equivalent procedure, in which $\mathrm{J}, \mathrm{W}$, and $\mathrm{Y}$ were predictive and $\mathrm{C}, \mathrm{M}$, and $\mathrm{V}$ were counterpredictive of target presence. During an experimental session, each subject was presented with either a random selection of nine lists from the same set (A or B) or eight randomly selected lists from one set and one final randomly selected list from the alternative set, as described below.

The result of this string-generation procedure was to create a correlation between the presence of certain nontarget letters and presence/absence of a target. For Stimulus Set A, the nontarget letters $\mathrm{C}, \mathrm{M}$, or $\mathrm{V}$ were thus relatively frequent among strings containing the target $S$, but relatively infrequent among target-absent strings. Js, Ws, and Ys were relatively frequent among target-absent strings (and relatively less frequent in strings containing a target). The letters $\mathrm{N}, \mathrm{O}$, and $\mathrm{R}$ were equally likely in either target-present or target-absent strings. However, no single letter or combination of letters predicted target presence with certainty, and individual letter strings varied in relative numbers of predictive and counterpredictive 


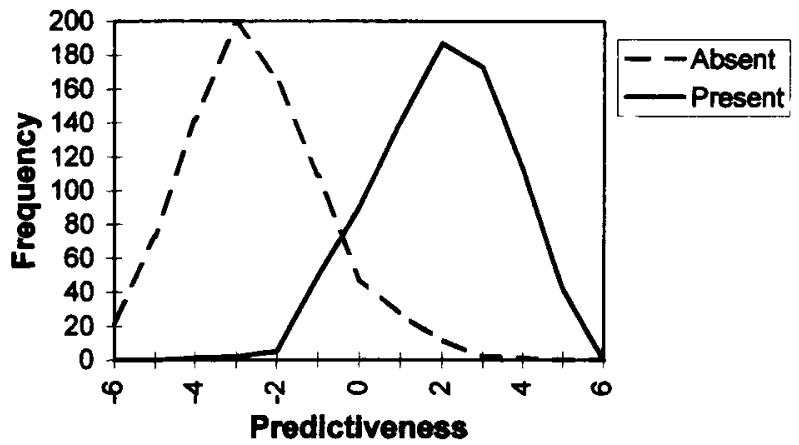

Figure 1. Frequency distributions of predictiveness values for target-present and target-absent strings across the 20 stimulus lists used in Set $A$.

letters. Furthermore, it should be noted that the particular sets of letters within the predictive, nonpredictive, and counterpredictive categories were selected to be quite heterogeneous in feature composition.

To illustrate the variability in relative numbers of predictive and counterpredictive nontarget letters in both target-present and target-absent strings, we computed a "predictiveness index" for each letter string in the stimulus lists, defined as the number of predictive letters minus the number of counterpredictive letters in a given string. For example, in Stimulus Set $\mathrm{A}$, the string CSOMMV contains a target and has a predictiveness of +4 , since it contains four predictive letters (two $\mathrm{Ms}$, a $\mathrm{C}$, and a $\mathrm{V}$, but no counterpredictive letters). The string $\mathrm{MC}$ SOYC has a predictiveness of +2 (three predictive letters- $M, C, C-$ and one counterpredictive letter-Y $-Y$. The target-absent string WCJJON has a predictiveness of -2 (three counterpredictive and one predictive letter). The frequency distributions of predictiveness values for target-present and target-absent letter strings across the 20 lists for Stimulus Set A are shown in Figure 1 (a highly similar distribution occurred for Stimulus Set B). From this distribution, it can be seen that the nontarget letters in some strings provided more predictive context than others, but that there was a moderately strong overall correlation between the predictiveness index and target presence.

Each string of six standard text font letters (white against black background) was presented individually on a 14-in. SVGA monitor until the subject responded. At the distance subjects were seated from the screen, the letter strings encompassed approximately $3^{\circ}$ of visual angle. A Microsoft QuickBasic program presented the stimuli. At the beginning of each trial block, a subject pushed a key when he/she was ready. A "get-ready" beep sounded approximately $500 \mathrm{msec}$ before each string was presented. The string of letters was then displayed in the center of the screen until the subject responded. Response key, response times, and information about the presented string (including target presence/absence and predictiveness index) were then written to a data file.

Procedure. The subjects were asked to indicate the presence or absence of a single target letter in each string by hitting one of two keys. Each subject was presented with nine trial blocks consisting of 80 trials each. For half of the subjects, the set of nontarget contingencies was reversed in the final trial block. That is, nontarget letters that had been positively correlated to the presence of a target were then negatively correlated with the presence of a target. The subjects were instructed to respond as rapidly as possible while avoiding errors.

Follow-up questionnaire. Following completion of all trial blocks, the subjects were given a questionnaire to assess several aspects of their memory for nontarget letters. Subjects who did not switch predictive information in the final trial block were first asked to write down each of the letters of the alphabet they had seen during the trial blocks (i.e., free recall of the nontarget letter set). These subjects were then shown, on the second page of the ques- tionnaire, 12 pairs of letter strings. In each pair, one string was consistent with the predictive information. A consistent string contained either a target, along with three predictive letters and two nonpredictive letters, or no target, along with three counterpredictive letters and three nonpredictive letters. The other string in the pair was in violation of the predictive information, pairing either a target with three counterpredictive letters and two nonpredictive letters or three predictive letters and three nonpredictive letters with no target. Subjects were asked to choose the string from each pair that "most closely resembled" the strings they had seen on the computer. The subjects were instructed to guess even if unsure. On the third page, the subjects were given three questions, each requiring them to choose which of three letters (one predictive, one nonpredictive, and one counterpredictive) they felt was most likely to have occurred in a string containing the target. Three equivalent questions were then given for strings not containing a target

Subjects who did switch predictive information in the final trial block were given a questionnaire with the same letter recall item as given the nonswitching subjects. The second page contained an item that required the subjects to rate the last trial block as being much easier than, somewhat easier than, about the same as, somewhat more difficult than, or much more difficult than previous blocks. The next item asked if the subjects had noticed any change in the composition or combinations of letters in the last trial block, as compared with earlier trial blocks. If the answer was "yes," the subject was asked to describe this change in his or her own words.

\section{Results and Discussion}

The first block of trials was considered practice for all subjects and was not included in the analyses. For each of the eight remaining experimental blocks, mean response times were calculated for each subject for targetpresent and target-absent trials. Response times greater than $1,000 \mathrm{msec}$ or less than $200 \mathrm{msec}$ were not included in the analyses. This resulted in exclusions of less than $1 \%$ of the trials. Pearson correlation coefficients between the "predictiveness" of a string (number of predictive letters minus number of counterpredictive letters) and response times were also calculated for each trial block. Subsequent analyses were based on these trial-block means. Three subjects from the switch condition were excluded from the analyses, 2 who had mean response times far slower than the remaining subjects in all blocks and 1 because of mechanical errors in data recording.

Response time analyses. Figure 2 displays mean response times across trial blocks, plotted separately for switching and nonswitching subjects. In target-absent trials, subjects for whom the predictive information changed in the final trial block showed increased reaction times for that block. The mean reaction time for the final trial block ( $573 \mathrm{msec}$ )' was $35 \mathrm{msec}$ longer than the average from the previous three blocks ( $538 \mathrm{msec})$, and this difference was statistically significant $[F(1,16)=10.76$, $\left.M S_{\mathrm{e}}=939.67, p<.01\right]$. In contrast, nonswitching subjects showed a slight decrease in reaction time $(18 \mathrm{msec})$ in the final block for target-absent trials, relative to the mean of the three previous blocks, but this difference was not statistically significant $\left[F(1,19)=1.63, M S_{\mathrm{e}}=360.88, p>\right.$ $.05]$. The performance decrement shown by switching subjects strongly suggests that the subjects were using the predictive information provided by the nontarget letters for trials in which the target was not present. 

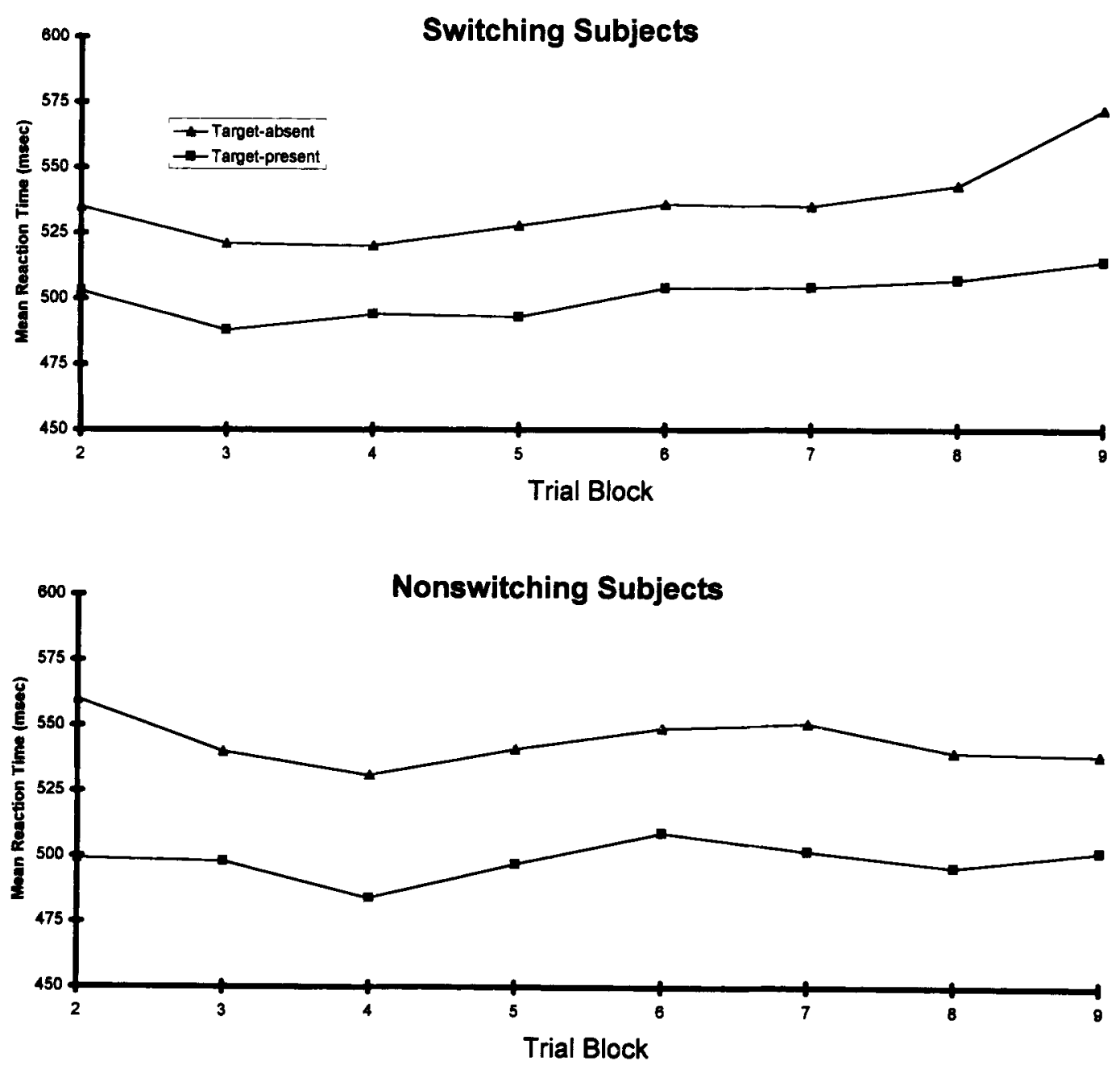

Figure 2. Mean reaction times across trial blocks for switching and nonswitching subjects in Experiment 1.

Although the "switching" subjects also showed a very slight increase in reaction time during the final trial block for target-present strings, this effect was not statistically significant $\left[F(1,16)=1.77, M S_{\mathrm{e}}=398.13\right]$. Furthermore, the "nonswitching" subjects showed a similar slight increase in reaction time in the final trial block. Thus, the presence of "counterpredictive" nontargets apparently facilitated "target-absent" responses more than "predictive" nontargets facilitated target detection.

Error analyses. Errors were infrequent in all trial blocks; subjects averaged less than $3 \%$ errors, and several subjects exhibited errorless performance in the majority of trial blocks. Therefore, error data were somewhat unstable. The proportion of errors in the final trial block (.03) was slightly, though not significantly, higher than in the previous three blocks (.02) for subjects who switched predictive information $[F(1,16)<1]$. Those who did not switch predictive information had an essentially identical proportion of errors in the last trial block (.02) as in the previous three.

Correlations between response time and predictiveness. As was shown in Figure 1, the predictiveness of both target-present and target-absent strings varied. Thus, addi- tional evidence concerning usage of the predictive context provided by the nontarget letters comes from an examination of Pearson's correlation coefficients between response time and the predictiveness index (number of predictive minus number of counterpredictive letters) in each individual letter string. If subjects were sensitive to the relative numbers of predictive and counterpredictive letters in each display, one would expect, for example, shorter response times to target-present strings containing a predominance of predictive letters. Similarly, shorter response times for target-absent trials would be expected for strings containing a predominance of counterpredictive letters. This should be observable for both nonswitching and switching subjects on the trials prior to the context switch.

To increase sensitivity, correlations were calculated by combining data from Trial Blocks 2-9 for nonswitching subjects and Blocks 2-8 (excluding the final trial block) for switching subjects. ${ }^{1}$ For target-present trials, the mean correlation between response time and predictiveness was -.058 for nonswitching subjects and -.062 for switching subjects (excluding data from the final block). These values are quite small, but have a negative sign con- 
sistent with the use of the context. Despite the small size of this correlation, it is important to note that 17 of 20 nonswitching subjects and 15 of 17 switching subjects showed a negative correlation.

For target-absent trials, the mean correlation between predictiveness and response time was +.136 for nonswitching subjects and +.123 for switching subjects (not including the final block), which is again in the expected direction and of slightly larger overall magnitude than the correlations observed for target-present trials. Correlations greater than zero were observed for all 20 nonswitching subjects and 16 of the 17 switching subjects. We did test for possible relationships between the size of these correlations and the response-time costs for switching subjects. No significant relationship was found (Pearson's $r$ relating the combined target-absent and target-present correlations for each subject to their switching costs was $-.272 ; d f=35 ; p>.05)$.

While the absolute magnitude of the correlations between predictiveness and response times are less than is typically considered of importance in behavioral research, the consistent pattern of correlation signs across subjects, which is significant by nonparametric sign test criteria for both target-absent and target-present trials, is evidence that subjects were sensitive to the information provided by the context of the nontarget letters. As with the switching costs in the response-time data, the effect appears to be greatest on target-absent trials. However, the predominance of negative correlations on target-present trials suggests that the contexts have had a small influence there as well, even though the comparison of pre- and postswitch response times did not reveal a significant effect.

Questionnaire analyses. Mean recall of nontarget letters was 2.2 (out of a possible 3.0 ) for predictive letters, 2.0 for nonpredictive letters, and 2.42 for counterpredictive letters. These values were not statistically different from one another $\left[F(2,78)=2.96, M S_{\mathrm{e}}=.61, p>.05\right]$, suggesting that subjects had no bias for recalling letters based on their predictive context. There were also no differences when these analyses were done separately for switching and nonswitching subjects. It should be noted that several subjects had perfect recall; thus, failure to observe potential differences may have been subject to ceiling effects in performance.

Analyses of the forced-choice item requiring nonswitching subjects to select which of two strings "most resembled" strings seen on the computer, showed an equal likelihood of choosing bias-consistent strings and bias-inconsistent strings. Two subjects were excluded from this analysis for failure to complete the questionnaire item. Of 12 pairs presented, the mean number "correct" (bias-consistent) was 6.0 , suggesting that subjects were responding to the stimuli randomly. Furthermore, the number of strings a subject identified correctly was not significantly related to their correlations between response time and amount of predictive information in either target-present $[r(16)=$ $.191, p>.05]$ or target-absent trials $[r(16)=.165, p>.05]$.

The items asking nonswitching subjects to indicate which of three letters occurred more frequently in target- present or target-absent strings were analyzed as threealternative multiple-choice items. The choice of a predictive letter was considered a correct response for the three items asking which letter had occurred more frequently in target-present strings, while the choice of a counterpredictive letter was considered the correct choice for the items asking about strings not containing a target. For these items, data from 6 subjects were excluded for failure to respond to every item. For the remaining 14 subjects, the mean number of correct responses was 1.08 for the items asking about target-present strings and 1.36 for the items asking about target-absent strings. Neither of these numbers differs significantly from the expected value of 1.0 (one third of three items). Furthermore, there was no relationship between the number of individual letters a subject correctly identified (Item 3 ) and the number of strings he or she correctly identified (Item 2) $[r(11)=-.01, p>$ $.05]$. Thus these patterns are not supportive of explicit knowledge or retention of a correlational rule or bias.

As a group, the subjects who switched contingencies in the final trial block reported that the final trial block had been of about the same difficulty as earlier trial blocks. Treated on a 5-point scale, where $1=$ much easier and $5=$ much more difficult, the mean rating of the difficulty of the final block was 3.3. This was not significantly different from the no-change-in-difficulty midpoint of 3.0 . Only 5 of 17 subjects reported that they noticed a change in the composition of the letters in the final trial block, and none of these 5 were able to verbalize anything similar to the actual structure of the predictive information. ${ }^{2}$ Collectively, these questionnaire analyses provide no evidence for either conscious retention of a correlational rule or explicit awareness of changes in task difficulty due to shifts in nontarget context.

\section{EXPERIMENT 2}

Experiment 1 showed that shifts in nontarget context could affect response time for target-absent trials but not for target-present trials. However, the weak, but consistent, correlations between nontarget context and response time in target-present trials suggest that nontarget context may have some small influence on performance. By requiring the subjects to search for multiple targets, thereby slowing overall response times on target-present trials and decreasing possible floor effects, it is possible that shifts in nontarget context could produce a noticeable cost in performance for both target-absent and target-present trials. For example, Flowers and Dutch (1976) demonstrated that in simple visual search for ink colors, the background context produced Stroop interference when subjects were required to search for three spectrally distributed ink colors, but the background context did not produce Stroop interference when subjects were required to search for only one or two ink colors. Such a pattern suggests that depth of processing nontarget information may depend upon the heterogeneity of the items in the target set. In Experiment 2, we sought to determine whether a similar memory load increase in a letter-search task would 

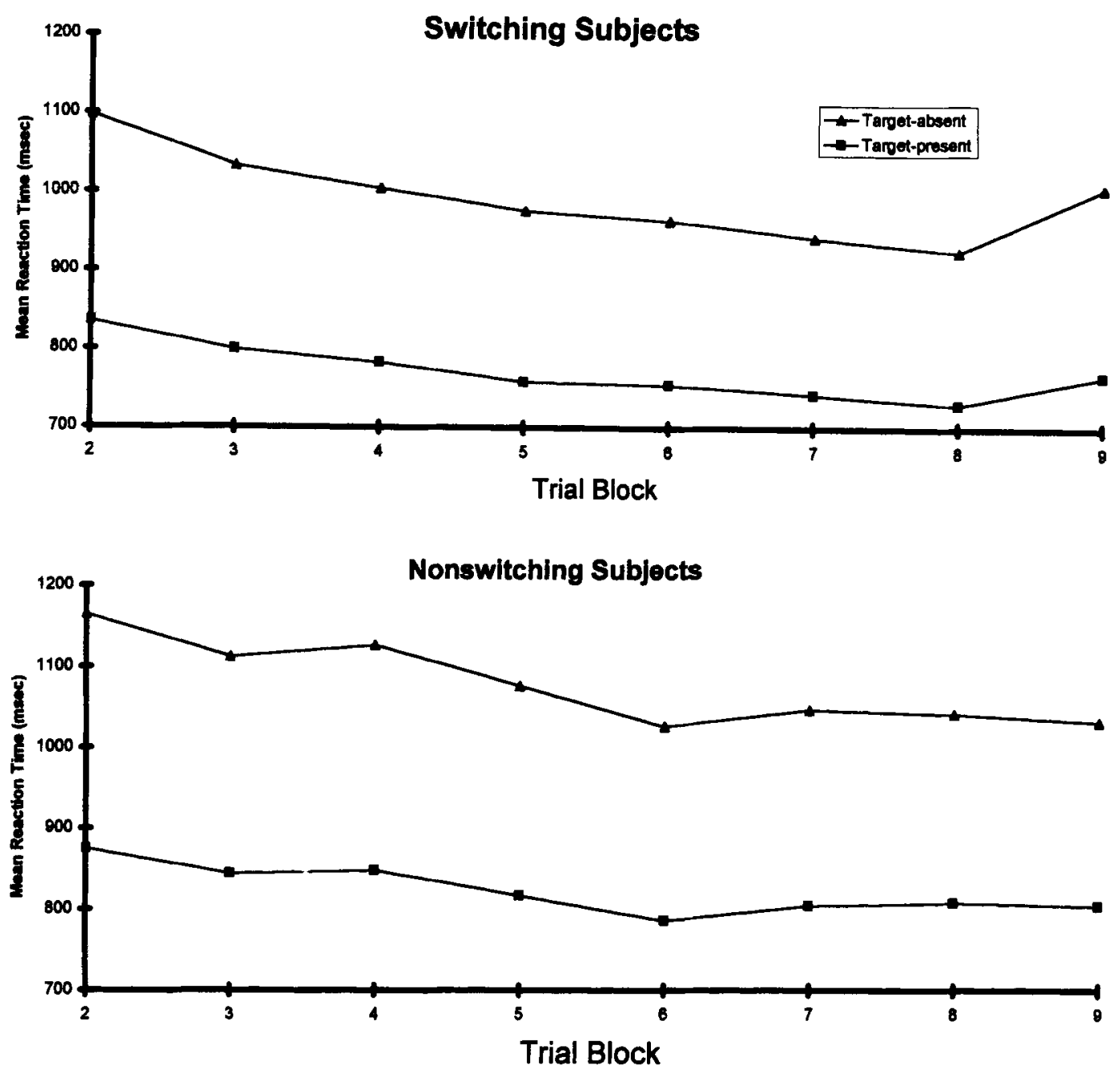

Figure 3. Mean reaction times across trial blocks for switching and nonswitching subjects in Experiment 2.

produce effects of predictive context of the nontarget letters in both target-present and target-absent trials.

\section{Method}

Subjects. Forty undergraduate students from an introductory psychology course participated in a single experimental session lasting approximately $1 \mathrm{~h}$. For participation, each subject received partial credit for a course requirement.

Stimulus materials. The stimulus materials of the second experiment were similar to those of the first except that there were three possible targets rather than a single one. New lists of stimuli were generated using the same computer-generation procedure and the same probabilities of predictive, nonpredictive, and counterpredictive letters in target-absent and target-present trials as were used in Experiment 1. However, in target-present strings, the stimulus-generation program randomly chose, with equal probability (.33), an " $\mathrm{H}$," a "P," or an " $\mathrm{S}$ " as the target letter. This task imposed a memory load of three items that substantially differed from one another in visual feature composition. No string contained more than one target letter.

Procedure. The procedure was essentially identical to that of Experiment 1, with the exception that the subjects responded "yes" by pressing the appropriate key whenever a letter string contained any one of the three target letters. Questionnaires with the same questions as those used in Experiment 1, though altered to reflect multiple targets, were also given.

\section{Results and Discussion}

The first block of trials was again considered practice for each subject and was not included in the analyses. For each subject, mean response times for each of the eight remaining trial blocks were calculated for target-present and target-absent trials. Response times longer than $1,500 \mathrm{msec}$ or shorter than $200 \mathrm{msec}$ were not included in the analyses. This exclusion criterion for long responses was greater than that of Experiment 1 due to the substantially longer response times required by a three-target search. Pearson correlation coefficients between the "predictiveness" of a string and response times were also calculated for each trial block. Subsequent analyses were based on these trial-block means. Also, one subject from the switch condition was excluded due to mechanical errors in recording the data.

Response time analyses. Figure 3 displays mean response time across trial blocks plotted separately for "switching" and "nonswitching" subjects. In general, re- 

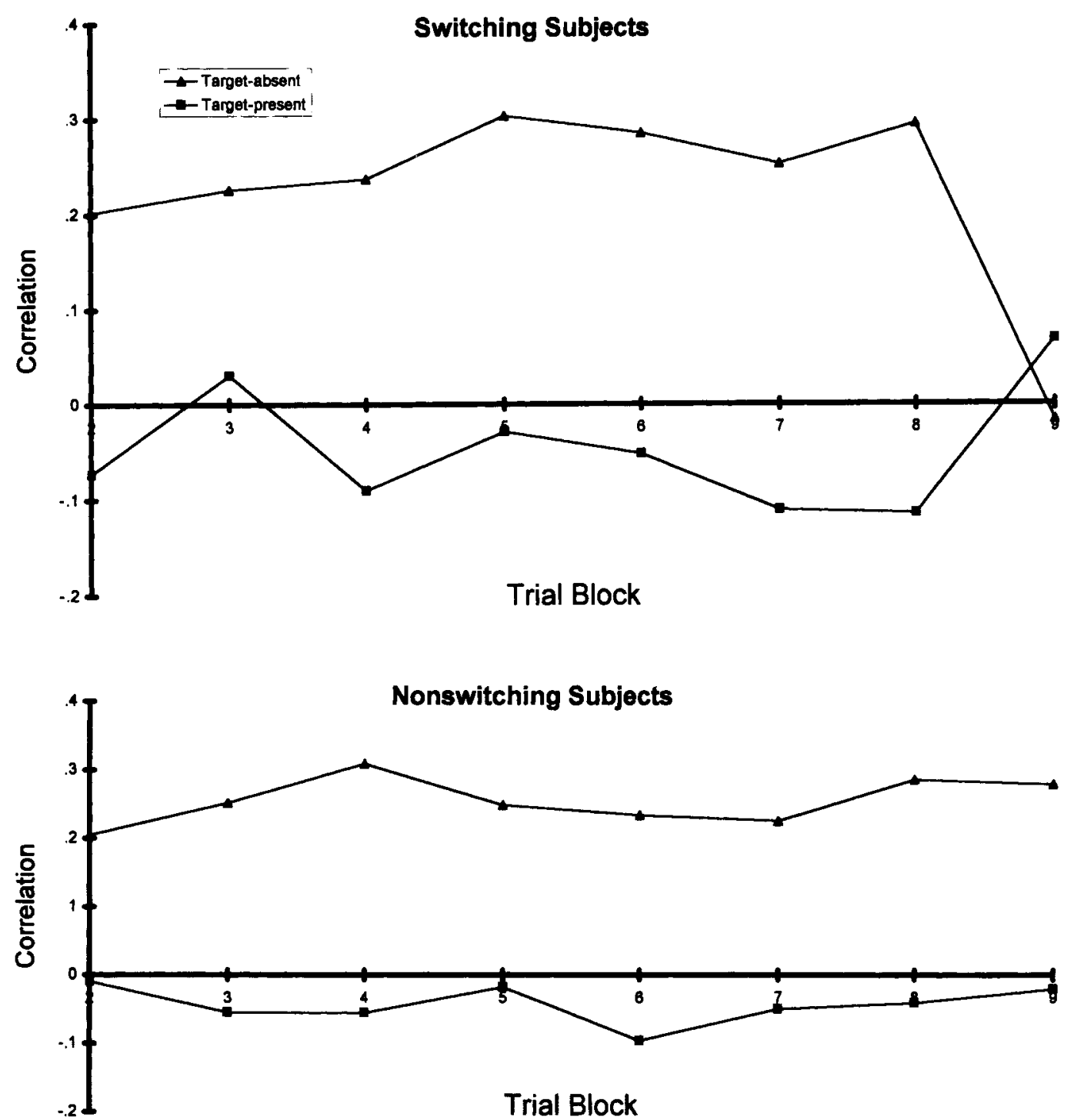

Figure 4. Correlations between mean reaction times and "predictiveness" of stimulus screens across trial blocks for switching and nonswitching subjects in Experiment 2.

action times decreased across trial block $[F(7,266)=$ $\left.12.94, M S_{\mathrm{e}}=5,221.92, p<.05\right]$. For target-absent trials, the subjects again showed an increase in response times (73 msec) on the final block as compared with the mean of the previous three trial blocks, and this effect was statistically significant $\left[F(1,18)=7.58, M S_{\mathrm{e}}=6,807.55\right.$, $p<.05]$. The subjects who did not switch predictive information in the final trial block showed no increase $(1,036$ vs. $1,037 \mathrm{msec}$ ). Unlike in Experiment 1, the switching of nontarget context also produced a significant $27-\mathrm{msec}$ response time cost for target-present trials $[F(1,18)=$ $\left.4.51, M S_{\mathrm{e}}=1,575.92, p<.05\right]$. Fourteen of 19 subjects had slower response time in the final trial block after the nontarget context changed.

Correlations between response time and predictiveness. Figure 4 illustrates correlations between response time and predictiveness of the nontarget context in a string, plotted separately for switching and nonswitching subjects and for target-absent and target-present trials. Because there was a general trend toward faster reaction times in later trial blocks, correlations were computed within trial blocks. Within target-absent trials, correlations between the predictiveness index of a string and reaction time (computed separately within each trial bock) averaged about .25 , a value larger than the overall correlation computed by aggregating the trial blocks for each subject in Experiment 1. All subjects, in both groups, produced a positive correlation in target-absent trials. Furthermore, switching subjects showed an attenuation of the correlations in the final block of trials relative to the previous three blocks [from +.29 to $-.0165 ; F(1,18)=24.44, M S_{\mathrm{e}}=.03, p<$ $.01]$, suggesting quite rapid "unlearning" of the predictive context. It should also be noted that, for both switching and nonswitching subjects, the correlation is greater than 
.20 even on the first experimental trial block (after the subjects had received only one practice block of 80 trials) and changed relatively little across the remaining blocks, supporting a relatively rapid acquisition process, as well. As in Experiment 1, correlations between the predictiveness of the string and response time were considerably lower in target-present trials, but were negative in sign for 16 of 19 switching subjects (not including the last trial block) and for 14 of 20 nonswitching subjects. As with the correlations for target-absent trials in switching subjects, there appears to be an attenuation of the correlations on the final block of trials; a statistical comparison of the mean correlation of .0682 for Block 9 with the average of -.0928 for the three previous blocks was statistically significant $\left[F(1,18)=4.51, M S_{\mathrm{e}}=6,835, p<.05\right]$. There was, however, no relationship between a subject's correlation and his or her response time costs when the predictive information was switched $[r(17)=.182, p>.05]$.

Error analyses. Errors were slightly more frequent in Experiment 2, averaging about 5\%, although several subjects had errorless performance on some trial blocks, and overall error rates were too low to separate into misses and false alarms. The switching subjects experienced an increase in error rates in the final block of trials (from $5.4 \%$ for the three blocks prior to the switch to $8.3 \%$ for the final block), and this increase was statistically significant $[t(18)=2.75, p<.05]$. Nonswitching subjects showed a nonsignificant decreasing trend for the equivalent comparison. Thus, error rates show costs in performance associated with context switch that mirror those obtained with response-time data.

Questionnaire analyses. For nonswitching subjects, mean recall of nontarget letters was 1.95 for predictive letters, 2.45 for counterpredictive letters, and 2.55 for nonpredictive letters. Unlike the data from Experiment 1 , these values were statistically different from one another $\left[F(2,38)=6.62, M S_{\mathrm{e}}=2.07, p<.01\right]$. The switching subjects, with corresponding recall values of 1.74 for predictive letters, 1.95 for counterpredictive letters, and 2.16 for nonpredictive letters, exhibited similar trends in that there was lower recall for the letter associated with targetpresent trials (predictive letters). One possible explanation of this effect concerns the faster, possibly self-terminating visual search process for target-present trials. Response times were about $200 \mathrm{msec}$ faster to target-present trials, in which predictive letters were concentrated, than to target-absent trials; thus subjects had less viewing time for, and perhaps devoted less attention to, nontargets in strings containing these letters. Alternatively, locating the target on target-present strings may result in the allocation of more attention to the target and less attention to the nontargets. ${ }^{3}$ Either of these interpretations also provides an additional explanation of why greater effects of nontarget context were observed on target-absent than on target-present trials. However, given that nontarget recall differences were not apparent in Experiment 1, caution is warranted concerning these interpretations.

As in Experiment 1, analyses of the forced-choice item requiring the nonswitching subjects to select which of two strings "most resembled" strings seen on the computer showed no significant tendency to select biasconsistent strings. Of 12 string pairs presented, the mean number of "correct" (bias-consistent) choices was 6.2. Furthermore, the number of strings a subject identified correctly was not significantly correlated with their correlation between response time and amount of background predictive information $[r(16)=-.054, p>.05]$.

For the items asking subjects to select which of three nontarget letters would have been more likely to occur in target-absent or target-present strings, 6 subjects were excluded from the analyses for failure to answer every item. The mean number of correct letters chosen was .93 for strings containing a target and 1.36 for strings not containing a target. The value of 1.36 approaches a statistically significant difference from the expected value of $1.0[t(13)=2.11, .1>p>.05]$. However, given that the recall questionnaire item given to the same subjects indicated greater ability to recall counterpredictive letters per se, it is difficult to interpret the results of this item as indicating explicit awareness of a rule versus a more general familiarity effect. Furthermore, there was no relationship between the number of individual letters a subject correctly identified (Item 3 ) and the number of strings he or she correctly identified (Item 2) $[r(12)=-.283, p>$ $.05]$, also indicating that there was little or no awareness of the structure of the background predictive information.

As a group, the subjects who switched contingencies in the final trial block were not likely to report that the final trial block had been more difficult than the earlier trial blocks. Treating this item on a 5-point rating scale, the mean was 3.05 , which did not significantly differ from the no-change-in-difficulty category of 3.0. For the final questionnaire item, 8 of 19 subjects reported that they had noticed a change in the composition of the letters in the final trial block, but none of them were able to verbalize something similar to the actual structure of the predictive information, suggesting, for example, that the position of the letter strings on the screen had changed or that the strings seen were the same as strings from earlier trial blocks. ${ }^{4}$ As with Experiment 1, these questionnaire analyses do not indicate retention or transfer of the perceptual learning revealed by actual task performance.

\section{GENERAL DISCUSSION}

Collectively, these two experiments have demonstrated that, in a simple visual search task, subjects are sensitive to the correlational structure between nontargets and the likelihood of target presence. Both costs in reaction time and increases in error rates occur when correlational structure is shifted. Furthermore, correlations between the amount of predictive context in individual letter strings and response times were observed within blocks of trials, and this pattern was noted even within the first experimental block of trials, suggesting that this form of learning occurs relatively rapidly. This kind of rapid acquisition is typical of related tasks which involve the learning of probabilistic relationships among events, such as the proba- 
bility learning tasks used by Reber and Millward (1968) and the flanker tasks used by Carlson and Flowers (1996) and Cohen and Shoup (1997).

Despite this evidence for rapid learning of targetnontarget associations, there was no evidence that subjects could use those associations as a basis to differentiate strings in the forced-choice task. Thus, while our stimuli resemble those of a concept-learning task in which subjects learn to discriminate between "s present" and "s absent" strings, or perhaps a form of simple but nondeterministic artificial grammar, our subjects were unable to perform the well-formedness or classification judgments typically used as criteria for learning in those experiments (much less express other evidence for explicit knowledge of stimulus structure). The associative learning involved in our task seems to resemble the sort of low-level association between visual form and response category that Miller (1987) attributes to nontarget probability effects in a flanker task.

There is another important feature of our data that is consistent with the view that what is learned is a lowlevel association between visual feature context and response priming. In contrast to the general finding that human beings are biased toward learning about positive incidences of events and are much less sensitive to negative or disconfirming information (e.g., Hearst, 1991), the correlation effects are most robust for target-absent trials. One would expect response priming effects to have the greatest effect for trials in which the perceptual decision processes are slower (e.g., tests for which multiple targets must be searched and trials on which an exhaustive search must be completed to determine target absence). In fact, this is the pattern that was found. Positive information bias may be more typical of active rulebased learning like that typically found in concept learning and other tasks for which learning of categorical structure is the primary objective.

The present findings also show that learning about correlations between targets and nontargets can occur in tasks that are more complex than simple flanker tasks. Such learning can clearly occur when correlations involve several different stimuli with quite heterogeneous features and for which displays contain multiple elements within a relatively cluttered display.

However, this study deals only with relative frequency of occurrence of context stimuli associated with the need to make a particular response. It is not clear whether similar effects would occur for more complex forms of correlated perceptual structure. For instance, would observers be sensitive to correlations between the presence of a specific pair of nontarget and target presence when no predictive information was provided by individual nontarget letters? Can subjects learn that displays containing red Xs and/or green Ts are more likely to contain a target while the presence of green Xs and red Ts is associated with target absence? Answers to questions such as these may provide insight about the nature of associative learning processes that allow the visual system to make use of context to optimize task performance.

\section{REFERENCES}

BERRY, D., \& DiENES, Z. (1993). Implicit learning: Theoretical and empirical issues. Hillsdale, NJ: Erlbaum.

Carlson, K. A., \& Flowers, J. H. (1996). Intentional versus unintentional use of contingencies between perceptual events. Perception \& Psychophysics, 58, 460-470.

COHEN, A., \& ShOup, R. (1997). Perceptual dimensional constraints on response selection processes. Cognitive Psychology, 32, 128-181.

Ericsson, K. A., \& Simon, H. A. (1980). Verbal reports as data. Psychological Review, 87, 215-251.

Eriksen, B. A., \& Eriksen, C. W. (1974). Effects of noise letters upon the identification of a target letter in a nonsearch task. Perception \& Psychophysics, 16, 143-149.

ERIKSEN, C. W., \& ST. JAMES, J. D. (1986). Visual attention within and around the field of focal attention: A zoom lens model. Perception \& Psychophysics, 40, 225-240.

FLOWERS, J. H., \& DUTCH, S. (1976). The use of visual and name codes in scanning and classifying colors. Memory \& Cognition, 4, 384-390.

HEARST, E. (1991). The psychology of nothingness. American Scientist, 79, 442-443.

MiLLER, J. (1987). Priming is not necessary for selective-attention failures: Semantic effects of unattended, unprimed letters. Perception \& Psychophysics, 41, 419-434.

ReBER, A. S. (1993). Implicit learning and tacit knowledge $\cdot$ An essay on the cognitive unconscious. New York: Oxford University Press. Reber, A. S., \& Millward, R. B. (1968). Event observation in probability learning. Journal of Experimental Psychology, 77, 317-327.

Treisman, A. M., \& Gelade, G. (1980). A feature-integration theory of attention. Cognitive Psychology, 12, 97-136.

YANTIS, S., \& JohNSTON. J. C. (1990). On the locus of visual selection? Evidence from focused attention tasks. Journal of Experimental Psychology: Human Perception \& Performance, 16, 135-149.

\section{NOTES}

1. While a statistically significant effect of trial block on mean response time across Blocks 2-8 was observed for target-absent trials $[F(6,222)=2.46, p<.05]$, no equivalent effect was found for targetpresent trials. Furthermore, Figure 2 suggests no apparent practice effect across Blocks 3-8. Thus, combining trial blocks for computation of the correlations appears justifiable.

2. The responses given by the subjects in Experiment 1 in attempting to explain the differences they had noted between the final trial block and earlier blocks were the following: "Fewer with the letter $S$ in it"; "More than one letter shown in the block and the blocks were ones from previous blocks"; "In the last trial block there were more Os and Cs"; "Arrangement of letters seemed different, earlier trial blocks S's seemed to be at the beginning or end"; "I thought the letters were more distinctit's harder to tell $\mathrm{C}+\mathrm{S}$ apart-I saw letters that were straight rather than curved."

3. The authors are indebted to Raymond Klein for this suggestion.

4. The responses given by the subjects in Experiment 2 in attempting to explain the differences they had noted between the final trial block and earlier blocks were the following: "repeated letters"; "It tended to throw me off by using letters similar to $P$ such as $R$ and similar [sic] $H$ such as $M$ and $N$ close to each other"; "I saw a lot [of] letter[s] V and $Y$ come up and they were very close to each other (more complex), and I can easily to [sic] tell the wrong letters such as $\mathrm{P}$ and H"; "It seemed like the letters $\mathrm{S}, \mathrm{H}, \mathrm{P}$ were either at the beginning of the string of [or] right at the end of the string"; "There [sic] were in different places on the screen and they were combined in a way that is [sic] was harder to tell which letter was which, ex. RMNNHV compared to earlier of YRSNM"; "more similar letters next to correct one"; "I think that in earlier trial blocks letters were coupled with the same letters, but in later blocks they did not seem [to be] so", "I don't remember seeing the letters V or J in the earlier trial blocks; I saw them in the last trial block."

(Manuscript received November 1, 1995; revision accepted for publication May 18, 1997.) 